\title{
Blood Flow Separator Design in Passive Lab-On-Chip Device
}

\author{
Sushmitha Maruthu Pandiyan, Kiruba Sebatiny Sebastin Solomon Kenned, Jeyasri Thenra, \\ Vigneswaran Narayanamurthy, Anisha. M, Anusha devi Kannan
}

\begin{abstract}
Nowadays, most of the clinical analytical tests are performed by separating the blood particles and it is exclusively used to diagnose the diseases in the medical field. There are various techniques which can be done through separating the particles, yet there are ways to go further for making the separation of particles efficient. Therefore, an on-chip integrated microfluidic device is required for separating the blood particles. The particle separation can be achieved by using porosity method which comes under the filtration techniques. The designed device consists of an inlet and an outlet reservoir. The device has a top channel and bottom channel for the blood flow where the filters are placed at the middle. By this way of filtration, it can easily separate normal and abnormal blood particles. From the whole blood sample, the particles are trapped by using hydrodynamics trapping method. The passive device is designed by COMSOL Multiphysics software and design results are presented.
\end{abstract}

Keywords — blood particles, blood flow separator, passive blood flow, filtration, hydrodynamics, microfluidics.

\section{INTRODUCTION}

Human blood consists of various components like RBC, $\mathrm{WBC}$, platelets. WBCs are larger in size than RBCs and platelets are the smallest blood components. These blood components are produced in the bone marrow [1]. Those components are used to diagnose diseases in clinical fields. The separation of plasma plays a vital role in the blood separation process. This enables to identify the differentiation of blood particles and abnormal cells. MEMS cater to the development of miniaturized devices. It has various domains which were proved by the revolutionary technology such as sensors, actuators, fluidics, optical MEMS etc [5]. It analyzes

Revised Manuscript Received on December 16, 2019.

Sushmitha Maruthu Pandiyan, Department of Biomedical Engineering, Kalasalingam Academy of Research and Education, Virudhunagar, Tamilnadu, India. Email: sushmitha.m@klu.ac.in

Kiruba Sebatiny Sebastin Solomon Kenned, Department of Biomedical Engineering, Kalasalingam Academy of Research and Education, Virudhunagar, Tamilnadu,India. Email: kirubasebatiny@gmail.com

Jeyasri Thenra, Department of Biomedical Engineering, Kalasalingam Academy of Research and Education, Virudhunagar, Tamilnadu, India. Email: jeyasrithenraj@ gmail.com

Vigneswaran Narayanamurthy, 1 Faculty of Electrical and Electronic Engineering Technology, Universiti Teknikal Malaysia Melaka, 76100 Durian Tunggal, Melaka, Malaysia. 2 Faculty of Electrical and Electronics Engineering, University Malaysia Pahang Pekan, Pekan, Pahang 26600, Malaysia. 3 Centre of Excellence for Advanced Research in Fluid Flow, University Malaysia Pahang, Kuantan 26300, Malaysia. Email: vigneswaran@utem.edu.my

Anisha. M, Department of Biomedical Engineering, Kalasalingam Academy of Research and Education, Virudhunagar, Tamilnadu, India. Email: anisha@klu.ac.in

Anusha devi Kannan, Department of Biomedical Engineering, Kalasalingam Academy of Research and Education, Virudhunagar, Tamilnadu, India. Email: anu.april211@gmail.com the minute volume of blood. Most of the MEM's device applications are used in the microfluidic device. It has its building blocks of the flow channel, pumps, needles, mixers, dispenses and reservoirs etc [3]. Macro devices ' primary disadvantage is that they need external mechanical sources such as pipe links between machines, syringes pumps, etc. Microstructure flows are therefore extremely preferred in straight channels [9]. The separation of particles can be performed using two methods: one is the active method and the other is the passive method [5]. Microchannels are intended to perform any fluid inside a fluid device for assessment. In some designs, the microchannel for dividing plasma has a large pressure loss by tight channels and can only flow very small quantities of samples per unit volume [2].

In the work reported by the particle separation utilizes the passive method. The particle separation has some physical properties such as size, density and cell deformability. Different methods are mentioned such as cross flow filtration, deterministic lateral displacement, centrifugation separation etc., $[5,21]$. In the work reported by for the continue flow of separation of particles, the Deterministic Lateral Displacement (DLD) is used. Compared to the other techniques, DLD is an effective method for particle separation. This technique is also based on the particle size, shape and its properties for the separation [9]. Micro fluidic devices have been developed for various application, such as biochemical, biomechanical, microelectronic etc. Blood particles separation is the important process in healthcare and clinical fields [6,7]. For regular examination of blood group of patients, the micro fluidic device will be very useful. This technique is the simplest and less expensive method. This process is achieved by reducing the amount of blood samples, analyzing time, using of bulk of apparatus [10].

Basically the micro fluidics device is manipulated in micro scale or nano scale object by applying various forces such as acoustic, electrical, dielectrophoresis, magnetic, optical forces etc. [1,8]. Particle separation can be done by different format like Compact Disc (CD), paper format but, it leads to high expenses regarding to the construction and establishment of the device. The separation of particles can be done with different filtration methods like weir, pillar, cross flow filter and silica microsphere filter [14,22,24]. Each method consists of various characteristics to separate the particles, and based on the samples, the choice of the method could be made. Cross flow filtration is used to remove large solid particles from the sample $[12,13]$. Weir filter is used to turn out cells free plasma. 


\section{Blood Flow Separator Design in Passive Lab-On-Chip Device}

Membrane filter filters the particles from plasma through pores. This is the porosity method by which the particles can be easily separated [22]. Separation of particles can also be done by the hydrodynamic methods. Micro devices consist of micro channel and filters, top channel, and bottom channel. By using of the mechanical force like syringe pump, the samples will be supplied into the device. In this device, there will be high efficiency, high throughput [11]. By using this device, the particles can be easily separated.

In the work reported, the membrane filtration method is used for the separation of various particles. It is also called as pressure technology. The particles get separated through their presence of semi-permeable membrane. In this work, hydrodynamic cell trapping method is used to make cells move along the filters and get laterally trapped in the bottom channel [16]. Using COMSOL Multiphysics, the microchannel has been designed using Computational fluid dynamics (CFD) module, simulated and analyzed [25]. In this paper, we propose many new designs using COMSOL and further discussions regarding the simulation results are typified for the separation of particles within the layers of different filters. The particle separation was carried out by manipulating the flow rate of channel's fluid flow and geometry of the bottom channel. From the simulation results, the separation of particles would be able to get trapped inside the bottom channel and efficient designs can be figured out. The modeling of the design and characterization is carried out by the COMSOL multi physics software [15,19]. In COMSOL software, the particle tracing method is used for simulating the results and laminar flow are mentioned here to trace the flow of the given sample. It is defined by the mathematical expression which varies from different parameters. COMSOL software are mainly used to design the channel with pores to analyze the flow behavior of velocity and pressure[26].

The blood particle separation is usually done by applying some of the external forces like electromagnetic forces, acoustic forces, dielectrophoretic forces, and optical forces [15]. There will be high fluidic resistance, low flow rate and particles get damaged. To overcome this in biological application, the low cost separation of particles without using any external forces are much needed [17].

\section{DEVICE DESIGN}

The proposed device consists of three filters with different pore sizes. Each filter can be used to separate the particles, depending upon the various size of the particles. In this device, it contains the top channel and bottom channel with inlet and outlet port. The whole size of the device is $75 \times 25 \mathrm{~mm}$ with $5 \mathrm{~mm}$ thickness as shown in Fig. 1 . Within this size, it consists of three filters. The pore size of the first filter is $11 \mu \mathrm{m}$ in diameter, which traps WBC. Normally the size of the $\mathrm{WBC}$ is about $12-17 \mu \mathrm{m}$. The pore size of the second filter is $5.5 \mu \mathrm{m}$ in diameter, which traps RBC. Its size is about $6-8 \mu \mathrm{m}$. The pore size of the third filter is $1.5 \mu \mathrm{m}$ in diameter, which traps the platelets. Its size is about $2-3 \mu \mathrm{m}$ [18]. By using these filters, the cells can be easily trapped due to their different morphology [20]. Finally, the other particle such as serum and plasma get filtered and it is taken out through the outlet. When the blood is made to flow through the inlet port, it passes to the filter by the top channel as shown in Fig.2, and the blood particles can be easily separated and also the abnormal particles can get trapped depending upon their size and shapes in their specified filters. These filter pores are designed with slight variations when compared with the normal particle size. The RBCs, WBCs and platelets get filtered separately and the plasma are collected in the bottom channel and gets out through the outlet [21].

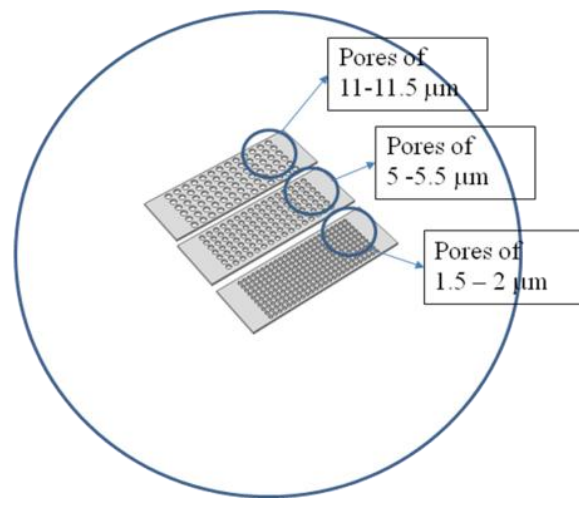

Fig. 1. Design of filters

TABLE I. DIMENSIONS OF DEVICE

\begin{tabular}{|l|l|l|}
\hline S.No & Design Description & Dimensions \\
\hline 1 & Length & $75 \mathrm{~mm}$ \\
\hline 2 & Width & $25 \mathrm{~mm}$ \\
\hline 3 & Depth & $5 \mathrm{~mm}$ \\
\hline 4 & $\begin{array}{l}\text { Gaps between } \\
\text { the filters }\end{array}$ & $0.625 \mathrm{~mm}$ \\
\hline 5 & $\begin{array}{l}\text { Thickness of the } \\
\text { filters }\end{array}$ & $0.5 \mathrm{~mm}$ \\
\hline
\end{tabular}

In COMSOL Multiphysics software to define the fluid flow like pressure and flow rate are used as physical qualities and also the viscosity and density are used as physical properties. According to Navier stokes equation the motion of the fluid flow is as expressed. The laminar flow is used for simulating fluid flows with high viscosity and geometrical length scales. It can also be used for time- dependent analysis [19]. From Fig.3,the mesh setting determines the resolution of finite elements analysis. It is used to discredit the model. In this model the Bernoulli's equation is used to the express the velocity and pressure for fluid flow, and according to this principle the velocity is inversely proportional to the pressure. The velocity is low, when the pressure is high. In biomedical application, high filtering efficiency can be acquired/obtained if the fluid- flow velocity low.

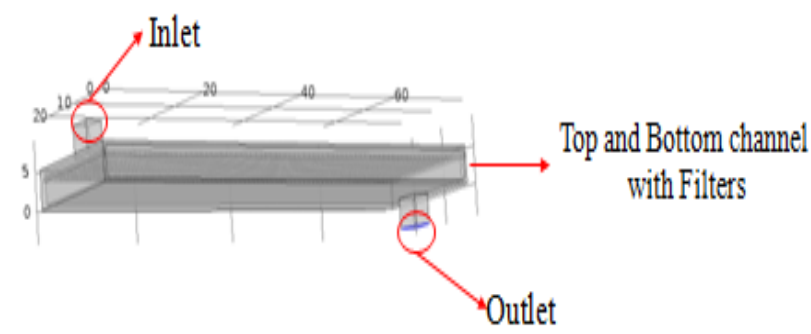

Fig. 2. Labelled parts

Published By:

Blue Eyes Intelligence Engineering \& Sciences Publication 


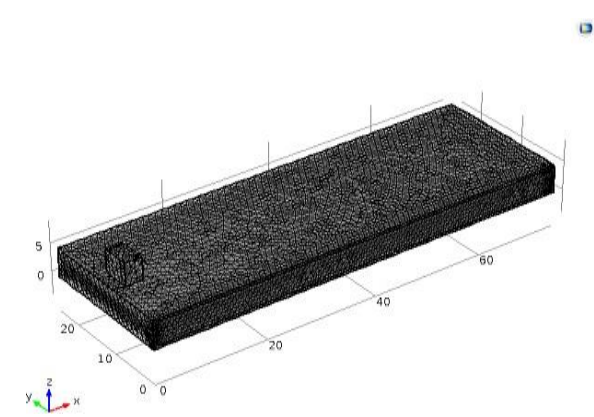

Fig. 3. Design after mesh processing

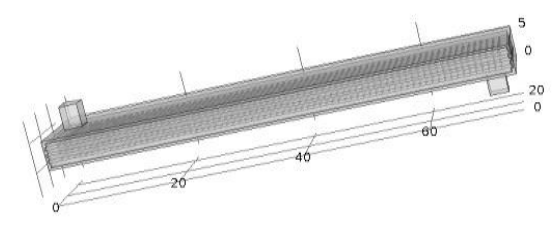

$\sum_{x \rightarrow x}^{2}$

Fig. 4. Design of device for particle separation

We initially designed three filters which can execute the flow of water and particles efficiently inside the filters. These filters have two channels such as top channel and bottom channel with single inlet and outlet, shown in Fig.4. The thickness of the filters ranges about $0.5 \mathrm{~mm}$. Particles size ranges between 4-17 micrometer. Water is considered as a buffer solution and is injected through the inlet of the channel. In a fluid flow behavior, to compute the motion of particles, particles-tracing is used. The boundary conditions shall include the selection of the design walls, the inlet, the outlet, and also the use of the pressure and velocity value in the particular boundary condition set, and shall predict the values in the fluid flow geometry. The fluid flow pattern considered is laminar flow. The particle trapping fluid behavior is also referred as stokes flow which occurs in systems with high velocity and length scales. The navier stokes equation preside over the motion of fluidics. It can be seen in fluidics as Newton's second laws of motion, and this includes.

$\rho(\partial \mathrm{u} / \partial \mathrm{t}+\mathrm{u} \cdot \nabla \mathrm{u})=-\nabla \mathrm{p}+\nabla \cdot\left(\mu\left(\nabla \mathrm{u}+(\nabla \mathrm{u})^{\wedge} \mathrm{T}-2 / 3 \mu(\nabla . \mathrm{u}) \mathrm{I}\right)+\mathrm{F}\right.$.

Where, velocity of fluid is ' $u$ '

pressure of fluid is ' $p$ ' density of fluid is ' $\rho$ '

dynamic viscosity of fluid is ' $\mu$ '.

These equations are solved for the continuity of fluid flow

$$
\partial \rho / \partial \mathrm{t}+\nabla \cdot(\rho \mathrm{u})=0 .
$$

The navier stokes equation corresponds to the conservation of momentum, and at the same time continuity equation corresponds to the conservation of mass. The navier stokes equation is for rectangular co-ordinates, from the naiver book, it is given as

$$
\rho \mathrm{Dv} / \mathrm{Dt}=\rho \partial \mathrm{v} / \partial \mathrm{t}+\rho(\mathrm{v} \cdot \nabla \mathrm{v})=-\nabla \mathrm{p}+\rho \mathrm{g}+\mu \nabla^{\wedge}(2) \mathrm{v} .
$$

\section{DESIGN AND SIMULATION ANALYSIS}

Substantial time derivative is defined as

$$
\rho \mathrm{Dv} / \mathrm{Dt}=\rho \partial \mathrm{v} / \partial \mathrm{t}+\rho(\mathrm{v} \cdot \nabla \mathrm{v}) .
$$

The hydrodynamic method was used to flow the fluid inside the microfluidic device. Depending upon the velocity and surface area of medium, hydrodynamic is applied to separate the particles.

$$
\mathrm{Fd}=1 / 2 \rho \vartheta^{\wedge} 2 \mathrm{Cd} \mathrm{A} .
$$

' $\mathrm{Fd}$ ' is the drag force,

' $\rho$ ' is the density of fluid, ' $\vartheta$ ' is the velocity of particles,

' $C$ ' and ' $d$ ' is the particles drag coefficient and area.

Among the above parameters, velocity and particles area can be measured using optical surveillance under microscope. Depending upon the particle's geometrical shape, the drag coefficient is dimensionless parameter. Reynolds number is very small for a microfluidics stream, i.e. the flow is fully laminar. At this low Reynolds number, the drag coefficient was optional for the spherical object as between 0.1-0.5.

To drag a particle inside microfluidics channel, two forces are required, Firstly, the Newtonian force and then the gravitational force.

$$
\mathrm{Fd}=\mathrm{Fn}+\mathrm{Fg}
$$

The particles will accelerate and push forward due to the drag force exerted on the particles. In our experimental conditions, i.e. the effect of gravity on the separation of particles at very low Reynolds number is very low and can be reduced.

As a result, Newtonian force can be equated with the drag force required to accelerate the particle.

$$
\mathrm{Fd}=\mathrm{Fn}=\mathrm{ma} \text {. }
$$

Where,

'Fn' is the force of exert ' $\mathrm{m}$ ' is the particle mass, and

' $a$ ' is acceleration due to the force.

The microfluidic device is airtight during fluid flow and therefore the pressure inside the micro filter is completely saturated, applying pressure inside the top channel is distributed evenly through the entire microfluidic device. Drag force on the surface of particles is equal to the force required to accelerate the particles at this point.

TABLE II. PARAMETERS FOR FLUID FLOW

\begin{tabular}{|c|c|r|c|}
\hline S.No & Description & Name & Expression \\
\hline 1 & Inlet velocity & u_in & $1 \mathrm{e}-4[\mathrm{~m} / \mathrm{s}]$ \\
\hline 2 & Fluid density & rho_f & $1000\left[\mathrm{~kg} / \mathrm{m}^{\wedge} 3\right]$ \\
\hline 3 & $\begin{array}{l}\text { Particle diameter: } \\
\text { WBCs }\end{array}$ & $\mathrm{dp} 1$ & $11[\mathrm{um}]$ \\
\hline 4 & $\begin{array}{l}\text { Particle diameter: } \\
\text { RBCs }\end{array}$ & $\mathrm{dp} 2$ & $5[\mathrm{um}]$ \\
\hline 5 & $\begin{array}{l}\text { Particle diameter: } \\
\text { Platelets }\end{array}$ & $\mathrm{dp} 3$ & $1[\mathrm{um}]$ \\
\hline
\end{tabular}




\section{Blood Flow Separator Design in Passive Lab-On-Chip Device}

\section{DESIGN SIMULATION RESUlTS}

Particle tracing method is discussed in the simulation results. It is calculated by the mathematical expressions of velocity and pressure [23]. The results are captured by the flow of water through the filters. It also induces the flow of blood for particle separation When the velocity gets increased the separation of the particle is takes place in the filter. The range varies from 0.001 to $1 \mathrm{~m} / \mathrm{s}$. by increasing the velocity it provides the unvarying flow of the sample diagonally the design. The maximum velocity acting on the field was recognized from fig 5 , and as shown in fig 6 , the filters are positioned at the precise stage. Furthermore, the graphical outcome for the separation of fluidic flow and cell particles demonstrates the elevated potential of the distinct filtering of blood cells.

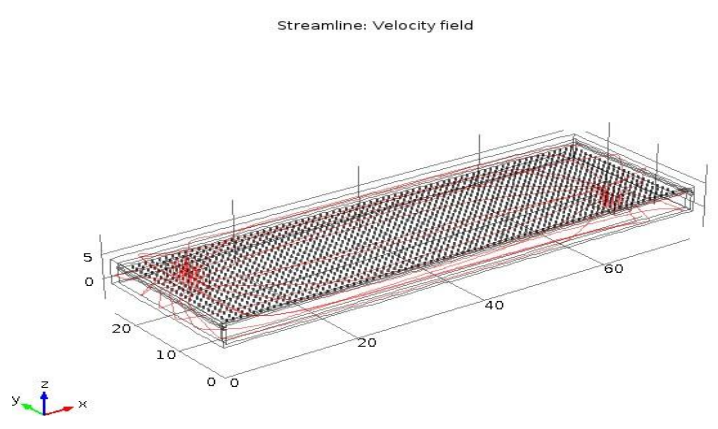

Fig. 5. Velocity field of fluid flow

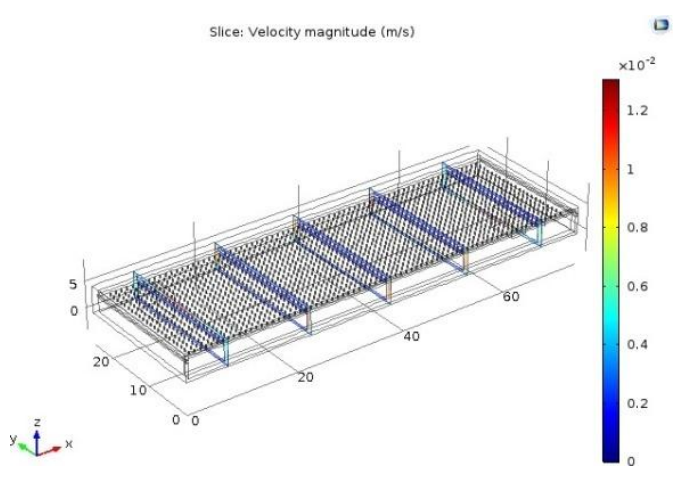

Fig. 6. Velocity magnitude of fluid flow

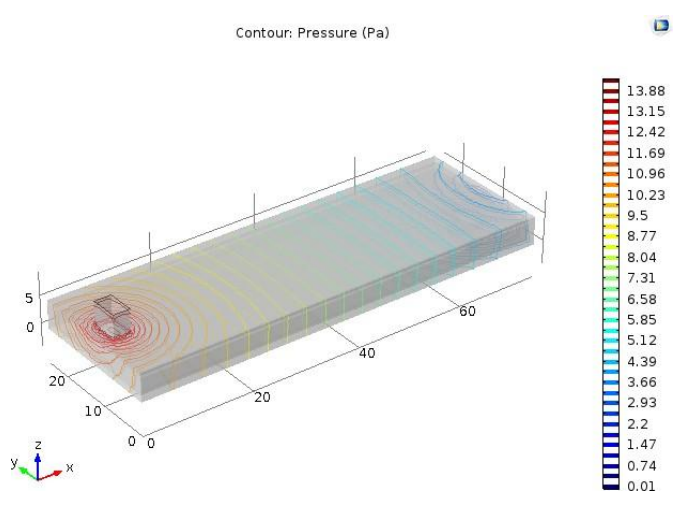

Fig. 7. Pressure along the device through fluid flow

\section{Conclusion}

The micro fluidic unit is therefore intended to separate blood particles. From the different simulation analyzes, we proposed that the instrument intended and the dimensions considered, as indicated in Table I, are relevant to the manufacture of a blood cell particle separation device with greater precision and high through place. The flow behavior of velocity and pressure can be analyzed in COMSOL software. In this device, we can separate the RBC, WBC, and platelets from whole blood and also the abnormal particles by using the filters. These particles get filtered according to their size and shapes. When all the particles are separated finally, the plasma can be moved out through the outlet which is fixed at the bottom channel. The purpose of designing this micro device is to separate the particles easily to diagnose the diseases with minimum amount of samples and also to reduce the time consumed to obtain the results. Experimentally and numerically calculated the separation efficiency of the designed microfluidic chip as determined by $95 \%$ of the targeted cells.

\section{REFERENCES}

1. Shamloo, Amir, and Farin Yazdan Parast. "Simulation of Blood Particle Separation in a Trapezoidal Microfluidic Device by Acoustic Force." IEEE Transactions on Electron Devices(2019).

2. Otsuki, H., T. Ota, and N. Miki. "Blood-Separating Device Without Energy Source for Implantable Medical Devices." In 2018 40th Annual International Conference of the IEEE Engineering in Medicine and Biology Society (EMBC), pp. 4661-4664. IEEE, 2018.

3. Faustino, Vera, Susana Catarino, Diana Pinho, Rui Lima, and Graça Minas. "A Passive Microfluidic Device Based on Crossflow Filtration for Cell Separation Measurements: A Spectrophotometric Characterization." Biosensors 8, no. 4 (2018): 125

4. Othamany, Nur Rabiatul Adawiyah Tajul, Norazreen Abd Aziz, Muhammad Izzuddin Abd Samad, Muhamad Ramdzan Buyong, and Burhanuddin Yeop Majlis. "Separation of Micro Engineered Particle Using Dielectrophoresis Technique." In 2018 IEEE International Conference on Semiconductor Electronics (ICSE), pp. 69-72. IEEE, 2018.

5. Omer, Saeed, Imran Suhyrani, and Yulin Deng. "Cell Separation Using Simple Microchip Configuration Experimental and Simulation Analysis." In 2017 10th International Symposium on Computational Intelligence and Design (ISCID), vol. 1, pp. 378-382. IEEE, 2017

6. Kumar, Praveen, T. Ramesh, and R. Aravind. "Silicon based bio-filter for bio-molecule separation." (2017).

7. Liu, Y., W. Dai, H. Li, W. Wu, and W. Wang. "A 3D filter for plasma separation from whole blood." In 2017 19th International Conference on Solid-State Sensors, Actuators and Microsystems (TRANSDUCERS), pp. 564-567. IEEE, 2017.

8. Saeed, O., and Yulin Deng. "Microdevice for magnetic cell separation simple fabrication and simulation analysis." In 2017 IEEE International Conference on Power, Control, Signals and Instrumentation Engineering (ICPCSI), pp. 2006-2010. IEEE, 2017.

9. Indhu, R., J. Anni Steffi Mercy, K. M. Shreemathi, S. Radha, S. Kirubaveni, and B. S. Sreeja. "Separation of bio-particles in micro fluidic device." In 2017 International Conference on Nextgen Electronic Technologies: Silicon to Software (ICNETS2), pp. 18-21. IEEE, 2017

10. Kan, Heng-Chuan, and Jian-Ming Lu. "Computational study of separation and capture of micro-particles in microfluidic devices." In 2016 International Conference on Advanced Materials for Science and Engineering (ICAMSE), pp. 677-679. IEEE, 2016.

11. Bazaz, Sajad Razavi, Ali Abouei Mehrizi, and Alireza Zabihi Hesari. "A novel microfluidic design for blood plasma separation." In 2016 23rd Iranian Conference on Biomedical Engineering and 2016 1st International Iranian Conference on Biomedical Engineering (ICBME), pp. 97-101. IEEE, 2016.

12. Lewpiriyawong, Nuttawut, and Chun Yang. "Dielectrophoresis field-flow fractionation for continuous-flow separation of particles and cells in microfluidic devices." In Advances in Transport Phenomena 2011, pp. 29-62. Springer, Cham, 2014.

13. Swargaloganathan, Divyagash. "Development of a cross flow filtration device for human blood cell fractionation." In 2014 40th Annual Northeast Bioengineering Conference (NEBEC), pp. 1-3. IEEE, 2014. 
14. Laki, András J., Gábor Zs Nagy, Kristóf Iván, Péter Fürjes, Olga Jacsó, and Pierluigi Civera. "Integrated microcapillary system for microfluidic parasite analysis." In 2013 IEEE Biomedical Circuits and Systems Conference (BioCAS), pp. 118-121. IEEE, 2013

15. Xue, Xiangdong, and Xueyong Wei. "Characteristics of blood flow in microchannels and relevant impact on modelling blood behaviour in biochip separators." In 2012 IEEE 62nd Electronic Components and Technology Conference, pp. 1547-1555. IEEE, 2012.

16. Seo, H. K., D. H. Kang, H. R. Ahn, H. O. Kim, and Y. J. Kim. "High throughput separation of blood cells by using hydrodynamics and magnetophoresis." In 2011 16th International Solid-State Sensors, Actuators and Microsystems Conference, pp. 1316-1319. IEEE, 2011.

17. Hou, Han Wei, Ali Asgar S. Bhagat, Wong Cheng Lee, Sha Huang, Jongyoon Han, and Chwee Teck Lim. "Microfluidic devices for blood fractionation." Micromachines 2, no. 3 (2011): 319-343.

18. Dong, Tao, Qianhua Su, Zhaochu Yang, Frank Karlsen, Henrik Jakobsen, Eirik Bentzen Egeland, and Snorre Hjelseth. "Fully integrated micro-separator with soft-magnetic micro-pillar arrays for filtrating lymphocytes." In 2010 Annual International Conference of the IEEE Engineering in Medicine and Biology, pp. 6522-6526. IEEE, 2010.

19. Xue, Xiangdong, Silvia Marson, Mayur K. Patel, Usama M. Attia, Chris Bailey, William O'Neill, David Topham, and Marc PY Desmulliez. "Biofluid behaviour in 3D microchannel systems: Numerical analysis and design development of 3D microchannel biochip separators." In 2010 Proceedings 60th Electronic Components and Technology Conference (ECTC), pp. 1021- 1030. IEEE, 2010.

20. Kavanagh, Deirdre M., Maïwenn Kersaudy-Kerhoas, Sumanth K. Pavuluri, Resham S. Dhariwal, and Marc PY Desmulliez. "Fabrication and testing of microfluidic devices for blood cell separation." (2009).

21. Zheng, Siyang, Jing-Quan Liu, and Yu-Chong Tai. "Streamline-based microfluidic devices for erythrocytes and leukocytes separation." Journal of Microelectromechanical systems 17, no. 4 (2008): 1029-1038.

22. Chang, Sunghwan, and Young-Ho Cho. "Continuous blood cell separation using a dielectrophoretic virtual pillar array." In 2008 3rc IEEE International Conference on Nano/Micro Engineered and Molecular Systems, pp. 974-977. IEEE, 2008

23. Khumpuang, S., T. Tanaka, F. Aita, Z. Meng, K. Ooe, M. Ikeda, Y. Omori et al. "Blood plasma separation device using capillary phenomenon." In TRANSDUCERS 2007-2007 International Solid-State Sensors, Actuators and Microsystems Conference, pp. 1967-1970. IEEE, 2007.

24. Han, Jungyoup, Se Hwan Lee, Youngmoo Heo, Chul Jin Hwang, and C. H. Ahn. "An on-chip blood serum separator using self-assembled silica microsphere filter." In The 13th International Conference on Solid-State Sensors, Actuators and Microsystems, 2005. Digest of Technical Papers. TRANSDUCERS'05., vol. 2, pp. 1688-1691. IEEE, 2005.

25. Narayanamurthy, Vigneswaran, Tze Lee, Al'aina Khan, Fahmi Samsuri, Khairudin Mohamed, Hairul Hamzah, and Madia Baharom. "Pipette Petri Dish Single-Cell Trapping (PP-SCT) in Microfluidic Platforms: A Passive Hydrodynamic Technique." Fluids 3, no. 3 (2018): 51

26. James Jasper, M. Sushmitha, R. Premkumar, Vigneswaran Narayanamurthy, and R. Kalpana. "Microfluidic micro-well (size and shape) by numerical optimization for single cell applications: Vertical trapping approach." In 2017 International conference on Microelectronic Devices, Circuits and Systems (ICMDCS), pp. 1-6. IEEE, 2017.

\section{AUTHORS PROFILE}

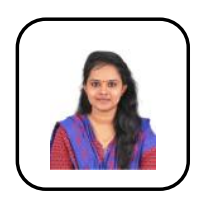

Ms. M. Sushmitha is currently working in the Department of Biomedical Engineering, Kalasalingam Academy of Research and Education. She had completed her Graduation, B.E in Engineering from Rajalakshmi Engineering College and Post-Graduation in Medical Electronics from Rajalakshmi Engineering College. She has published her research papers in various IEEE international conferences and one journal.

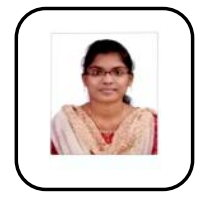

Ms. S. Kiruba Sebatiny is currently working in a Biomedical Company. She had completed her Graduation, B.Tech in Engineering from Kalasalingam Academy of Research and Education. She has done project in Foot Neuropathy Analayser For Diabetic Ulcer.

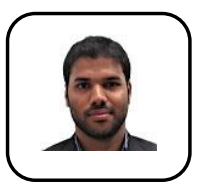

Dr. Vigneswaran Narayanamurthy is an academician cum researcher with several years of experience in research, teaching and consultancy in the field of Electronics and Biomedical Engineering. Currently he is serving as senior lecturer in the faculty of electrical and electronic engineering technology, Universiti Teknikal Malaysia Melaka. Have published in several indexed international journals and holds two patents. Products developed through past research include a passive lab on chip applications, expert systems, and agriculture technologies. 